\title{
Vaccination anti-typhique.
}

Les succès constatés dans les armées des Indes et des Etats-Unis par l'emploi des vaccins antityphiques attirent de plus en plus l'attention des services de santé des armées et la méthode semble devoir se généraliser. Au Japon, ainsi, 28.000 sujets déjà ont été vaccinés et, sur ce nombre, la morbidité a été réduite de 14 à 1 et la mortalité de 2,4 à 1 . Aux Etats-Unis, pendant les dix-huit derniers mois, plus de 43.000 soldats ont été vaccinés contre cette maladie et ils n'ont fourni que 11 cas de fièvre typhoïde qui tous ont guéri. Dans la division mobilisée au Texas, sur 15.000 hommes on ne compte, en quatre mois, que 2 cas de fièvre typhoïde. Or, sous la même latitude, en 1898, en quatre mois aussi, une division, composée de 10.759 hommes compta 1729 cas avérés de fièvre typhoïde avec 248 décès.

De tels faits dispensent de commentaires et actuellement la preuve est faite de l'efficacité de ce moyen prophylactique ; il ne reste guère qu'à déterminer quels sont, parmi les vaccins employés, ceux dont les résultats sont les meilleurs. Quant à la diffusion de la méthode, d'après un résumé de M. le Dr Granjux dans le Caducée ${ }^{1}$ sur "l'état actuel de la question de la vaccination antityphoïdique ", celle-ci n'aurait guère, jusqu'ici, franchi les frontières des armées anglaises, américaines et japonaises. En Allemagne, toutefois, elle fut pratiquée pendant le transport des troupes dans le sudouest africain en 1905. Dans la plupart des pays d'Europe et leurs colonies, par contre, cette vaccination n'est usitée ni dans la population civile ni dans l'armée. On sait pourtant la gravité et la fréquence relative de certaines épidémies de fièvre typhoïde, surtout dans les garnisons et campagnes coloniales.

En France, où l'on a proposé de remplacer le vaccin de Wright par ceux de Chantemesse et de Vincent, les expé-

${ }^{1}$ No 2, 1912, p. 19. 
riences ne datent guère que de quelques mois et les vaccinations ne sont que de quelques centaines, tandis que c'est par centaines de mille qu'elles se comptent déjà dans les colonies anglaises.

On peut dire qu'actuellement les oppositions, nombreuses encore et parfois irréductibles il n'y a pas longtemps, s'évanouissent devant les faits, surtout en ce qui concerne l'armée. Souhaitons que partout les faits confirment les heureux résultats constatés et que cette mesure prophylactique, si précieuse contre la maladie la plus redoutable des armées en campagne, donne à l'avenir tout ce qu'on est déjà en droit d'en attendre.

$D^{\mathbf{F}} \mathrm{F}$.

\section{GRÈCE}

\section{Relations officielles entre la Groix-Rouge et l’armée}

Les relations officielles entre la Croix-Rouge hellénique et l'armée grecque, sont réglées par le décret royal en date du 25 novembre 1910. Ce décret prescrit, dans ses articles 212-217, quelles doivent être les relations officielles entre l'armée d'une part et la Croix-Rouge hellénique d'autre part, de même que les rapports avec les míssions des Sociétés étrangères de secours aux blessés militaires et malades ou avec toute autre société poursuivant le même but.

Voici la traduction de ces articles 212-217 de ce

\section{Règlement du service sanitaire en campagne}

Sociétés de secours aux blessés de la guerre

Art. 212. - En cas de guerre, la Croix-Rouge hellénique, les missions des Croix-Rouges étrangères et toute autre Société ont le droit d'aménager des hôpitaux temporaires et infirmeries de gare, des navires-hôpitaux, des trains sanitaires ainsi que des hôpitaux permanents dans l'intérieur. 